\title{
A Preliminary Investigation into the Association of Chloride Concentration on Morbidity and Mortality in Hospitalized Canine Patients
}

This article was published in the following Dove Press journal: Veterinary Medicine: Research and Reports

\section{Madeline B Libin (ID) Joel G Weltman (iD Jennifer Prittie}

Department of Emergency and Critical Care, Animal Medical Center, New York, NY, USA
Correspondence: Madeline B Libin Department of Emergency and Critical Care, Animal Medical Center, 510 E. 62nd Street, New York, NY 10065, USA

$\mathrm{Tel}+\mid 2128388100$

Fax + I 2127522592

Email madelinelibin@gmail.com
Purpose: To evaluate whole blood chloride concentration and hospital-acquired AKI in hospitalized canine patients. Secondary outcome measures included the volume-adjusted chloride load, in-hospital mortality and length of ICU stay.

Patients and Methods: This is a prospective, observational study. Sixty dogs admitted to the ICU and receiving IV fluid therapy for $>24$ hours from February 2018 to July 2019. Corrected chloride and creatinine concentrations were obtained twice daily. Total volume of IV fluid and total chloride load were recorded. Volume-adjusted chloride load (VACL) was calculated by dividing the chloride administered by the volume of fluid administered. Hospital-acquired AKI was defined as an increase in creatinine of $\geq 26.5 \mu \mathrm{mol} / \mathrm{L}(0.3 \mathrm{mg} / \mathrm{dL})$ or $150 \%$ from baseline to maximum. Survival to hospital discharge or non-survival and ICU length of stay were also recorded.

Results: Fifteen out of 60 patients developed hospital-acquired AKI. Maximum corrected chloride was significantly different in AKI group (median $122.3 \mathrm{mmol} / \mathrm{L}$ ) vs non-AKI group (median $118.1 \mathrm{mmol} / \mathrm{L} ; \mathrm{p}=0.0002$ ). Six out of 60 patients developed hyperchloremia. Hyperchloremic patients were significantly more likely to develop in-hospital AKI $\left(\mathrm{p}=0.03\right.$ ). Patients hospitalized $\geq 2$ days had a significantly higher $\left[\mathrm{Cl}^{-}\right]_{\max }$ compared to those with shorter ICU stay $(121.8 \pm 5.9 \mathrm{mmol} / \mathrm{L}$ vs $117.5 \pm 4.3 \mathrm{mmol} / \mathrm{L} ; \mathrm{p}=0.002)$. Eight out of 60 patients were non-survivors. Maximum corrected chloride and creatinine concentrations were not significantly different between survivors and non-survivors. VACL was not significantly different between AKI or mortality groups.

Conclusion: Maximum corrected chloride concentration was significantly higher in dogs with hospital-acquired AKI, even amongst dogs without hyperchloremia. Additionally, maximum corrected chloride concentrations were significantly higher in dogs hospitalized in the ICU longer compared to those hospitalized for fewer than two days. There was no significant difference in VACL in any of the outcome groups. Results from this study suggest alterations in chloride may be observed alongside the development of acute kidney injuries. Future studies in critically ill dogs are warranted.

Keywords: acute kidney injury, IV fluid, electrolytes, hyperchloremia, volume-adjusted chloride load

\section{Introduction}

The administration of intravenous chloride is ubiquitous in hospitalized veterinary patients and chloride concentration is routinely measured in veterinary hospital practice and ICU settings. This electrolyte has recently received attention as a potential mediator of morbidity and mortality in critically ill human and veterinary patients. 
Serum chloride disturbances are commonly observed in critically ill dogs with diverse disease etiologies. ${ }^{1,2}$ While increased mortality has been related to serum chloride disturbances in dogs, ${ }^{3,4}$ it remains unclear if this is reflective of the severity of primary underlying disease processes or if chloride plays an inherent role in disease progression. A recent abstract presented at the 2016 International Veterinary Emergency and Critical Care symposium found that the presence and severity of hospitalacquired hyperchloremia and hypochloremia in dogs and cats were associated with higher fatality rates than community-acquired chloride disturbances ${ }^{4}$ suggesting that inhospital interventions may play a role in the morbidity and mortality related to dyschloremia in these species. In human studies, it has been suggested the use of supraphysiologic intravenous chloride solutions, such as $0.9 \%$ saline (normal saline; NS), carries an increased risk of acute kidney injury (AKI), mortality, prolonged ICU stay, and hyperchloremic metabolic acidosis as compared to balanced crystalloid solutions. ${ }^{5-7}$ While these findings may suggest that alterations in chloride concentration are associated with increased morbidity and mortality in sick veterinary patients, at this point in time it is not known whether it is the chloride itself, the volume of IV fluid administered, or a combination of factors may be associated with poorer outcomes reported.

A large retrospective study in human patients with SIRS attempted to determine the effect of chloride alone as a potential primary driver of deleterious consequences associated with crystalloid fluid administration. Utilizing a calculation called the volume-adjusted chloride load (mmoL of administered chloride/liters of intravenous fluid administered; VACL), the investigators demonstrated a significant positive correlation between mortality and VACL during fluid resuscitation in humans fulfilling SIRS criteria. ${ }^{8}$ This finding supports the notion that the absolute volume of chloride administered is influential on patient outcomes. To the authors' knowledge, there are no veterinary studies investigating the specific volume of chloride administered and morbidity or mortality in critically ill dogs or cats.

The primary objective of the present study is to evaluate the association between corrected chloride concentration and hospital-acquired AKI in hospitalized canine patients. Secondary outcome measures included association of VACL and in-hospital mortality and length of ICU stay (LOS) and association between corrected chloride concentration and in-hospital mortality and LOS. We hypothesized that elevations in corrected chloride concentration and VACL would be associated with hospitalacquired AKI and mortality.

\section{Patients and Methods}

Dogs consecutively admitted to the emergency department of a large, urban, private hospital between March 2018 and August 2019 were eligible for this study. The study protocol was approved by the Institutional Animal Care and Use Committee and informed client consent was obtained prior to study enrollment. Inclusion criteria were admission to the ICU and administration of greater than 24 hours of IV crystalloid fluid therapy. Dogs receiving antihypertensive medication, dogs previously diagnosed with chronic kidney disease as categorized by historic measurement of creatinine $>123.8 \mu \mathrm{mol}(1.4 \mathrm{mg} / \mathrm{dL})$ prior to hospitalization, or previous or intended diuretic therapy including furosemide, spironolactone, and/or angiotensinconverting enzyme inhibitors were excluded from the study. Dogs were also excluded if they had received treatment with IV fluids prior to presentation, had missing data, or were euthanized, discharged, or moved to a general hospitalization ward less than 24 hours following enrollment. Data recorded for each study participant at the time of emergency presentation included signalment, diagnosis, weight, heart rate, and rectal temperature.

Blood pressure measurements were obtained in $60 \%$ $(35 / 60)$ of dogs. Systolic and mean arterial pressures were obtained in 25/35 dogs using an oscillometric monitor (Midmark Corporation, Dayton, OH, USA). The remainder of dogs (10/35) had systolic blood pressure measured with a Doppler monitor (Parks Medical Electronics, Inc., Las Vegas, NV, USA). All blood pressure measurements were obtained on a peripheral limb utilizing a cuff size that was approximately $40 \%$ of the limb circumference.

Venous blood (between 0.2 and $0.4 \mathrm{~mL}$ ) was collected anaerobically into a heparinized syringe at the time of peripheral catheter placement and analyzed via cage side biochemistry analyzers. Baseline (T0) peripheral venous chloride and creatinine concentrations were measured (i-STAT Chem 8. Abbot Point of Care Inc. for Abaxis Inc. Union City, CA, USA). Venous blood gas analysis and lactate measurement (Siemens Rapid Point 500, Malvern, PA, USA) were also performed. Complete blood counts (IDEXX CBC, IDEXX Laboratories, Westbrook, ME, USA) and serum chemistry panels (IDEXX Catalyst One, IDEXX Laboratories, Westbrook, ME, USA) were also obtained prior to admission to the ICU. Venous sodium, chloride, 
and creatinine concentrations (i-STAT Chem 8. Abbot Point of Care Inc. for Abaxis Inc. Union City, CA, USA) were obtained every 12 hours during ICU hospitalization either by direct venipuncture or via sampling catheter using standard collection techniques. $^{9}$

Every four hours following ICU admission, total volume of IV solutions administered during the previous four hours (inclusive of all continuous rate infusions, bolused solutions, low-volume saline drug diluent and heparinized saline flushes) were recorded on an integrated ICU treatment and study sheet. Volumes documented were inclusive of medications (including additional diluent), isotonic and hypertonic crystalloid solutions, potassium chloride supplementation, blood products, and synthetic and natural colloids. Volumes of water or food administered via nasogastric or esophageal tubes were not recorded. Patients that had procedures under general anesthesia (including surgery or diagnostic imaging) were included and volumes of IV solutions administered during anesthesia were documented. Chloride of administered plasma was not available; however, as plasma was commercially acquired, an assumed normal chloride of $120 \mathrm{mmol} / \mathrm{L}$ was used for these products. All data were gathered for the first 72 hours of ICU hospitalization. All patient data required for the study were extracted by the authors from the patients' electronic medical records and laboratory computerized results.

Variables collected for analysis included maximum measured corrected chloride $\left([\mathrm{Cl}]_{\max }\right)$, maximum measured creatinine $\left([\mathrm{Cr}]_{\max }\right)$, maximal change in corrected chloride concentration (difference between peak and baseline), total volume of IV fluid infused during hospitalization, and total chloride load (summing of chloride content for all IV fluid solutions). For the purposes of this study, hyperchloremia was defined as a blood chloride concentration of $>126 \mathrm{mmol} / \mathrm{L}$ based on the reference interval (106-126 mmol/L) established by the manufacturer of the cage side analyzer (i-STAT Chem 8. Abbot Point of Care Inc. for Abaxis Inc. Union City, CA, USA). The chloride values utilized for all calculations were those corrected for sodium utilizing the following equation: $\left[\mathrm{Cl}^{-}\right]_{\text {corrected }}=$ $\left(\left[\mathrm{Na}^{+}\right]_{\text {normal }} /\left[\mathrm{Na}^{+}\right]_{\text {measured }} \mathrm{x}\left[\mathrm{Cl}^{-}\right]_{\text {measured }}\right)$, where normal $\mathrm{Na}^{+}$for dogs was $146 \mathrm{mEq} / \mathrm{L} .{ }^{10}$ Chloride load was adjusted for fluid volume received by dividing total mmol chloride by total fluid volume. This measure, expressed in $\mathrm{mmol} / \mathrm{L}$, is referred to as volume-adjusted chloride load (VACL). Volume-adjusted chloride load was also expressed based on bodyweight as VACL per kilogram of bodyweight (VACL/kg).

Twice daily creatinine concentrations obtained for each patient were used to document the development of hospital-acquired AKI defined as an increase in blood creatinine of $\geq 26.5 \mu \mathrm{mol} / \mathrm{L}(0.3 \mathrm{mg} / \mathrm{dL})$ or $150 \%$ from baseline to maximum. ${ }^{11}$ The reference interval for creatinine concentration established by the manufacturer of the cage side analyzer is $44.21-114.95 \mu \mathrm{mol} / \mathrm{L}(0.5-1.3 \mathrm{mg} / \mathrm{dL})$ (i-STAT Chem 8. Abbot Point of Care Inc. for Abaxis Inc. Union

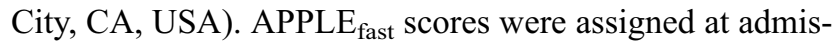
sion and calculated based on patient glucose, albumin, lactate, platelet count and mentation score. ${ }^{12}$ Survival to hospital discharge or non-survival (death or euthanasia), as well as LOS, were also recorded.

\section{Statistical Methods}

Prior to study commencement, a power analysis was performed to determine minimum sample size to detect a difference in the development of AKI with progressive hyperchloremia. Utilizing an effect size of 0.85 (moderate effect) and significance level of 0.05 , a sample size of 10 dogs per group was estimated to find a significant effect for hyperchloremia on AKI. The previous reported incidence of hospital-acquired AKI in dogs is approximately $12 \%{ }^{13}$ and therefore a sample size of 60 was deemed appropriate.

A Shapiro-Wilk test was performed to assess for normality of data. All descriptive data following parametric distribution are expressed as mean and standard deviation whereas those following non-parametric distribution are expressed as median and range. For the purposes of primary and secondary outcome analyses, animals were grouped based on four different outcomes: the development of AKI or not; the development of hyperchloremia or not; LOS $\geq 2$ days or $<2$ days; and in-hospital survival or non-survival. Dogs that were euthanized or died were not evaluated separately due to low number of participants in these groups. Comparisons between continuous variables were performed by Wilcoxon rank-sum test or Student's $T$-test as appropriate based on distribution. Comparisons between categorical variables were performed by Fisher's exact test. A p-value $<0.05$ was deemed significant. All analyses were performed on commercial statistical software (Stata SE 15.1, StataCorp, College Station, TX).

\section{Results}

Eighty-eight dogs were enrolled into the study. A total of 28 were missing data or remained in the ICU less than 24 
hours following enrollment and were excluded. Thus, 60 patients were eligible for further evaluation (Figure 1). Of the remaining 60 participants, 32 were male neutered, 5 were male intact, 19 were female spayed, and 4 were female intact. Demographics and baseline characteristics of the entire study population are shown in Table 1 .

The most common disease processes were acute hemorrhagic diarrhea syndrome (6/60), trauma $(5 / 60)$, aspiration pneumonia (4/60), lymphoma (4/60), complications related to diabetes mellitus (4/60), extrahepatic biliary duct obstruction $(3 / 60)$, anemia $(2 / 60)$, gastrointestinal foreign bodies $(2 / 60)$, cervical myelopathy $(2 / 60)$, and gastric dilatation $(2 / 60)$.

Fifty-six out of 60 patients received PlasmaLyte A (Baxter Healthcare Corporation, Deerfield, IL) as the primary crystalloid resuscitation fluid during hospitalization; 2/ 60 patients received lactated Ringer's solution (Baxter Healthcare Corporation, Deerfield, IL); 2/60 patients received a solution prepared by combining equivalent volumes of LRS and either sterile water or sterile water with 5\% dextrose. Assessment for differences in expected proportions in fluid administration between outcome groups was not possible due to inadequate sample size for analysis (Table 2).
Fifteen out of 60 patients developed AKI (AKI group) during the period of hospitalization. The mean systolic blood pressure at presentation was significantly higher in dogs developing in-hospital AKI $(161.5 \mathrm{mmHg} \pm 30.8)$ vs those that did not develop AKI $(131.5 \mathrm{mmHg} \pm 33.8)(\mathrm{t}=-2.5$, $\mathrm{p}=0.02$, Table 3 ). There were no significant differences in the remainder of baseline characteristics between AKI groups (Table 3 ). The maximum corrected chloride was significantly different in the hospital-acquired AKI group (122.3 mmol/L (range 117-133.4)), vs the non-AKI group (118.1 mmol/L (range 109-139.5) $)(z=-3.7, p=0.0002$, Table 3, Figure 2)

Over the course of the study period, $6 / 60$ (10\%) patients developed hyperchloremia. No differences in baseline characteristics were found between those that developed hyperchloremia and those that did not (Table 4). Of these, 4/6 developed hospital-acquired AKI. While the maximum creatinine was no different between patients which developed hyperchloremia $(110.53 \mu \mathrm{mol} / \mathrm{L}$, range $61.89-557.05 \mu \mathrm{mol} /$ $\mathrm{L} ; 1.25 \mathrm{mg} / \mathrm{dL}$, range $0.7-6.3 \mathrm{mg} / \mathrm{dL}$ ) vs those which did not (75.16 $\mu \mathrm{mol} / \mathrm{L}$, range 35.37-221.05 $\mu \mathrm{mol} / \mathrm{L} ; 0.85 \mathrm{mg} / \mathrm{dL}$, range $0.4-2.5 \mathrm{mg} / \mathrm{dL} ; \mathrm{z}=-1.4, \mathrm{p}=0.16$, Table 4 ), those that developed hyperchloremia were significantly more likely to develop an in-hospital AKI ( $p=0.03)$.

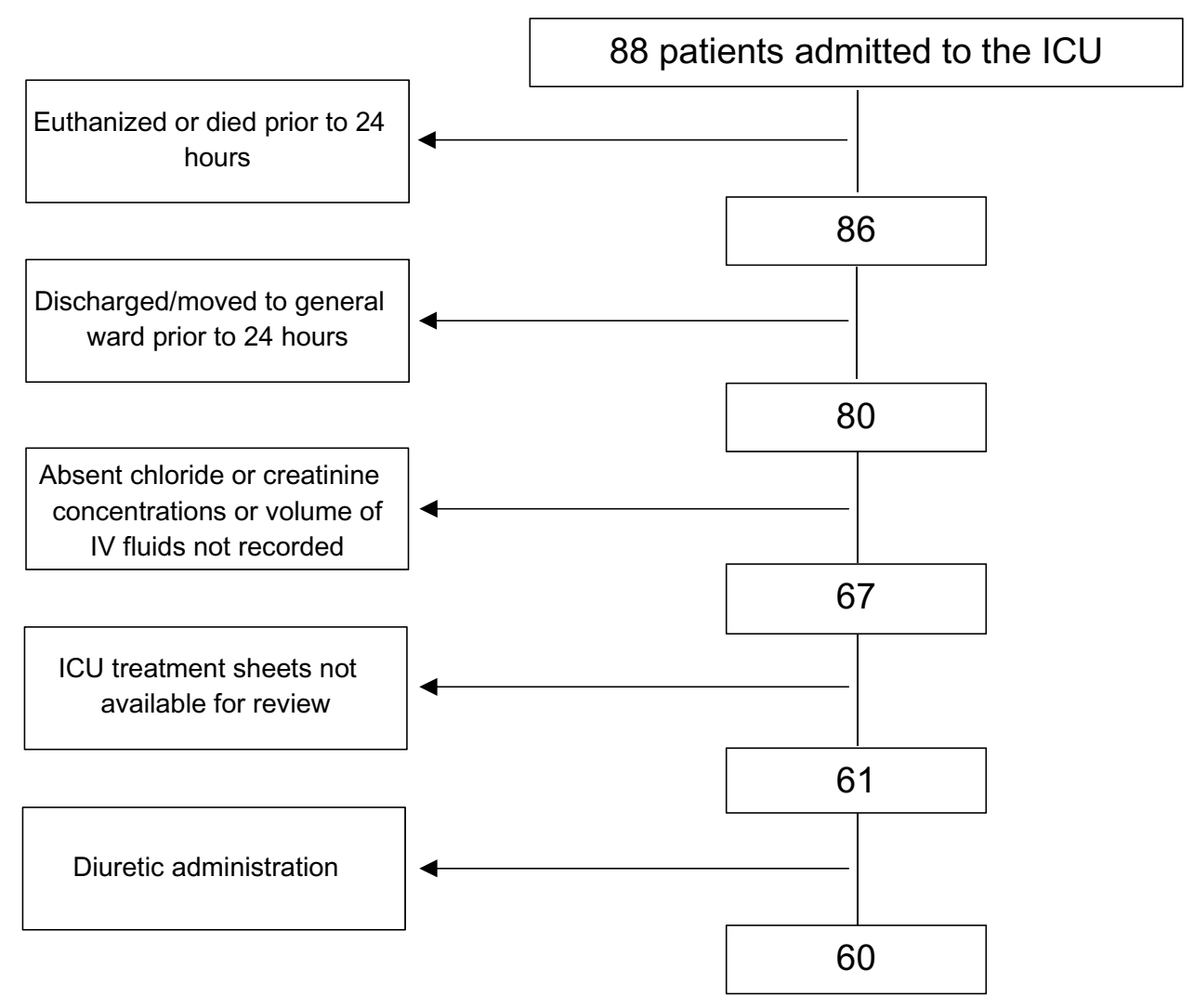

Figure I Flowchart of patients enrolled and excluded in the study. 
Table I Summary Statistics for Total Study Population. Values are Presented as Mean and Standard Deviation for Normally Distributed Data and Median with Range for Non-Normally Distributed Data

\begin{tabular}{|c|c|}
\hline Characteristics & $\begin{array}{l}\text { Total Population } \\
(n=60)\end{array}$ \\
\hline Age (years) & $8(0.67-15.5)$ \\
\hline Weight (kg) & $9.75(2-43)$ \\
\hline Heart rate (beats $/ \mathrm{min}$ ) & $139 \pm 34$ \\
\hline Mean temperature $\left({ }^{\circ} \mathrm{C}\right)$ & $38.5 \pm 1.2$ \\
\hline $\mathrm{SBP}(\mathrm{mmHg})$ & $|4| \pm 35.4(n=35)$ \\
\hline MAP (mmHg) & $114 \pm 31.6(n=25)$ \\
\hline APPLE $_{\text {fast }}$ score (range) & $20(\mathrm{I}-37)$ \\
\hline$\left[\mathrm{Cl}_{\text {corr }}\right]$ on presentation $(\mathrm{mmol} / \mathrm{L})$ & $110.0 \pm 5.73$ \\
\hline$\left[\mathrm{Cl}_{\text {corr }}\right]_{\max }(\mathrm{mmol} / \mathrm{L})$ & II8.8 (109-139.5) \\
\hline$\Delta_{\operatorname{MAX}}\left[\mathrm{Cl}_{\text {corr }}\right](\mathrm{mmol} / \mathrm{L})$ & $9.95(1-35.8)$ \\
\hline$[\mathrm{Cr}]$ on presentation $(\mu \mathrm{mol} / \mathrm{L})$ & $70.74(26.53-433.26)$ \\
\hline$(\mathrm{mg} / \mathrm{dL})$ & $0.8(0.3-4.9)$ \\
\hline$[\mathrm{Cr}]_{\max }(\mu \mathrm{mol} / \mathrm{L})$ & $79.58(35.37-557.05)$ \\
\hline$(\mathrm{mg} / \mathrm{dL})$ & $0.9(0.4-6.3)$ \\
\hline Fluid $_{\text {tot }}(\mathrm{mL})$ & $949(115.5-14,397)$ \\
\hline Fluid $_{\text {tot }} / \mathrm{kg}(\mathrm{mL} / \mathrm{kg})$ & IIII.0 (21.0-334.8) \\
\hline $\mathrm{Cl}_{\text {tot }}^{-}(\mathrm{mmol})$ & $106.6(19.5-1718.0)$ \\
\hline VACL (mmol/L) & $0.11(0.06-0.21)$ \\
\hline $\begin{array}{l}\text { No. of patients receiving synthetic colloids } \\
\text { (\%) }\end{array}$ & $9 / 60(15 \%)$ \\
\hline $\begin{array}{l}\text { No. of patients receiving fresh frozen } \\
\text { plasma (\%) }\end{array}$ & $1 / 60(1.67 \%)$ \\
\hline No. of patients receiving albumin (\%) & $4 / 60(6.67 \%)$ \\
\hline
\end{tabular}

Abbreviations: SBP, systolic blood pressure; MAP, mean arterial pressure; [ $\left[\mathrm{Cl}_{\text {corr }}\right]$, corrected chloride concentration; $\left[\mathrm{Cl}_{\mathrm{corr}}\right]_{\max }$, maximum corrected chloride concentration; $\Delta_{\text {MAX }}\left[\mathrm{Cl}_{\text {corr }}\right]$, maximum change in corrected chloride concentration; [Cr], creatinine concentration; $[\mathrm{Cr}]_{\max }$, maximum creatinine concentration; Fluid $\mathrm{tot}_{\text {to }}$ total fluid administered $(\mathrm{mL})$; Fluid $\left.\mathrm{tot}_{\mathrm{tog}} / \mathrm{kg} / \mathrm{mg}\right)$, total fluid administered per kilogram; $\mathrm{Cl}_{\text {tot }}^{-}$, total chloride delivered; $\mathrm{VACL}$, volume-adjusted chloride load.

Twenty-six participants remained in the ICU for two days or more. Baseline blood pressures were significantly lower in those with longer ICU hospitalization times (systolic: $127 \mathrm{mmHg}$, range $70-181$ vs $142 \mathrm{mmHg}$, range
Table 3 Demographic and Patient Clinical Data Classified by Development of AKI. Values are Presented as Mean and Standard Deviation for Normally Distributed Data and Median with Range for Non-Normally Distributed Data

\begin{tabular}{|c|c|c|c|}
\hline \multirow[t]{3}{*}{ Characteristics } & \multicolumn{2}{|l|}{ AKI } & \multirow[t]{3}{*}{ p-value } \\
\hline & Yes & No & \\
\hline & $n=15$ & $n=45$ & \\
\hline Age (years) & $8.5(I-15)$ & $7.5(0.67-15.5)$ & 0.75 \\
\hline Weight $(\mathrm{kg})$ & $17.8(2.0-42.2)$ & $8.9(2.64-43)$ & 0.22 \\
\hline $\begin{array}{l}\text { Heart rate } \\
\text { (beats } / \mathrm{min} \text { ) }\end{array}$ & $149 \pm 30$ & $136 \pm 34$ & 0.21 \\
\hline Temperature $\left({ }^{\circ} \mathrm{C}\right)$ & $38.8 \pm 1.3$ & $38.3 \pm 1.2$ & 0.2 \\
\hline $\mathrm{SBP}(\mathrm{mmHg})$ & $161.5 \pm 30.8(n=11)$ & $\begin{array}{l}|3| .5 \pm 33.8 \\
(n=24)\end{array}$ & $* * 0.02$ \\
\hline $\operatorname{MAP}(\mathrm{mmHg})$ & $130 \pm 33.8(n=7)$ & $\begin{array}{l}107.7 \pm 29.3 \\
(n=18)\end{array}$ & 0.12 \\
\hline APPLEfast score & $20(13-26)$ & $20(1-37)$ & 0.9 \\
\hline $\begin{array}{l}{\left[\mathrm{Cl}_{\text {corr }}\right] \text { on }} \\
\text { presentation } \\
(\mathrm{mmol} / \mathrm{L})\end{array}$ & $1 \mathrm{II} .7 \pm 4.04$ & $109.5 \pm 6.13$ & 0.20 \\
\hline $\begin{array}{l}{\left[\mathrm{Cl}_{\mathrm{corr}}\right]_{\max }} \\
(\mathrm{mmol} / \mathrm{L})\end{array}$ & $122.3(\mid 17.0-133.4)$ & $118.1(109-139.5)$ & $* * 0.0002$ \\
\hline $\begin{array}{l}{[\mathrm{Cr}] \text { on }} \\
\text { presentation } \\
(\mu \mathrm{mol} / \mathrm{L}) \\
(\mathrm{mg} / \mathrm{dL})\end{array}$ & $\begin{array}{l}44.21 \\
(26.53-433.26) \\
0.5(0.3-4.9)\end{array}$ & $\begin{array}{l}79.58 \\
(35.37-2 \mid 2.2 \mathrm{I}) \\
0.9(0.4-2.4)\end{array}$ & $* * 0.007$ \\
\hline $\begin{array}{l}{[\mathrm{Cr}]_{\max }(\mu \mathrm{mol} / \mathrm{L})} \\
(\mathrm{mg} / \mathrm{dL})\end{array}$ & $\begin{array}{l}97.26 \\
(61.89-557.05) \\
\text { I.I (0.7-6.3) }\end{array}$ & $\begin{array}{l}70.74 \\
(35.37-221.05) \\
0.8(0.4-2.5)\end{array}$ & $* * 0.01$ \\
\hline
\end{tabular}

Note: **Denotes $p$-value of significance $(p<0.05)$.

Abbreviations: SBP, systolic blood pressure; MAP, mean arterial pressure; $\left[\mathrm{Cl}_{\mathrm{corr}}\right]$, corrected chloride concentration; $[\mathrm{Cr}]$, creatinine concentration; $[\mathrm{Cr}]_{\max }$, maximum creatinine concentration; $\left[\mathrm{Cl}_{\text {corr }}\right]_{\max }$, maximum corrected chloride concentration.

90-205; $\mathrm{z}=2.27, \mathrm{p}=0.02 ;$ MAP: $89.5 \mathrm{mmHg}$, range 67-160 vs $120 \mathrm{mmHg}$, range $86-174 ; \mathrm{z}=2.67, \mathrm{p}=0.01$, Table 5). Patients remaining in the ICU for 2 or more days had a higher median baseline creatinine concentration (88.42 $\mu \mathrm{mol} / \mathrm{L}$, range $35.37-433.26 \mu \mathrm{mol} / \mathrm{L} ; 1.0 \mathrm{mg} / \mathrm{dL}$,

Table 2 IV Fluid Administration Classified by Outcome Group

\begin{tabular}{|l|l|l|l|l|l|l|l|l|}
\hline \multirow{2}{*}{ IV Fluid Product Administered } & \multicolumn{2}{l}{ AKI } & \multicolumn{2}{l|}{ Hyperchloremia } & \multicolumn{2}{l|}{ Length of Stay } & \multicolumn{2}{l|}{ Mortality } \\
\cline { 2 - 9 } & Yes & No & Yes & No & $\geq \mathbf{2}$ days & $<2$ days & Death & Survival \\
\cline { 2 - 9 } & $\mathbf{n = 1 5}$ & $\mathbf{n = 4 5}$ & $\mathbf{n = 6}$ & $\mathbf{n = 5 4}$ & $\mathbf{n = 2 6}$ & $\mathbf{n = 3 4}$ & $\mathbf{n = 8}$ & $\mathbf{n = 5 2}$ \\
\hline P-lyte A (n=56) & $13 / 15$ & $43 / 45$ & $5 / 6$ & $51 / 54$ & $22 / 26$ & $34 / 34$ & $8 / 8$ & $48 / 52$ \\
LRS (n=2) & $0 / 15$ & $2 / 45$ & $0 / 6$ & $2 / 54$ & $2 / 26$ & $0 / 34$ & $0 / 8$ & $2 / 52$ \\
1/2 LRS (n=2) & $2 / 15$ & $0 / 15$ & $1 / 6$ & $1 / 54$ & $2 / 26$ & $0 / 34$ & $0 / 8$ & $2 / 52$ \\
VS (n=9) & $3 / 15$ & $6 / 45$ & $0 / 6$ & $9 / 54$ & $7 / 26$ & $2 / 34$ & $4 / 8$ & $5 / 52$ \\
FFP (n=I) & $0 / 15$ & $1 / 45$ & $0 / 6$ & $1 / 54$ & $1 / 26$ & $0 / 34$ & $1 / 8$ & $0 / 52$ \\
Canine albumin (n=4) & $1 / 15$ & $3 / 45$ & $0 / 6$ & $4 / 54$ & $4 / 26$ & $0 / 34$ & $2 / 8$ & $2 / 52$ \\
\hline
\end{tabular}

Abbreviations: P-lyte A, PlasmaLyte A; LRS, lactated Ringer's solution; 1/2 LRS, half-strength lactated Ringer's solution; VS, VetStarch; FFP, fresh frozen plasma. 


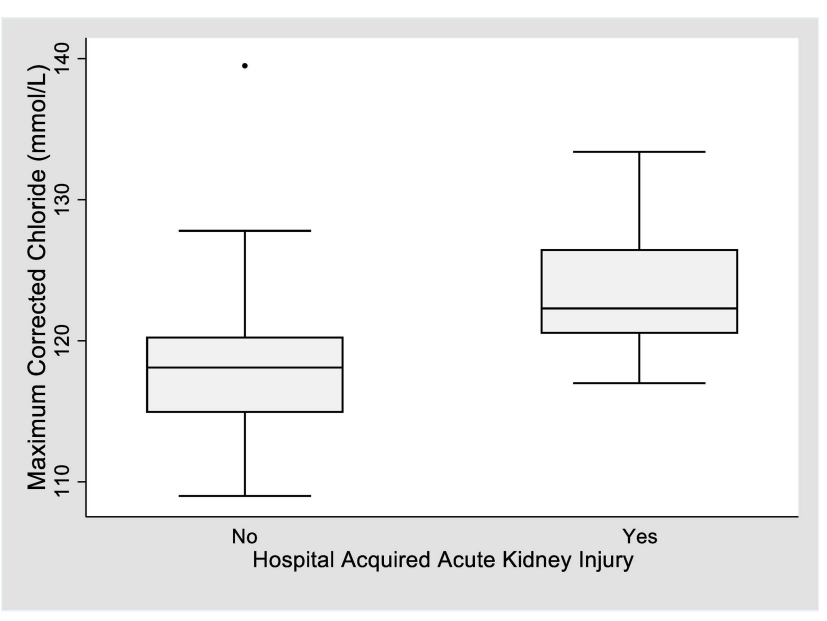

Figure 2 Box and whisker plot of maximum corrected chloride concentration between AKI groups.

Notes: Boxes represent the interquartile range from the 25 th to the 75 th percentile. The horizontal bar in each box represents the median value. The T-bars represent the range. The black dots represent outliers. The reference interval for chloride is $106-126 \mathrm{mmol} / \mathrm{L}$. The median maximum corrected chloride concentration was significantly higher in the AKI group (I $22.3 \mathrm{mmol} / \mathrm{L}$; range II7-133.4 $\mathrm{mmol} / \mathrm{L})$ as compared to the non-AKI group (118.1 mmol/L; range 109-139.5 $\mathrm{mmol} / \mathrm{L})(p=0.0002)$

range $0.4-4.9 \mathrm{mg} / \mathrm{dL}$ ) compared to those with shorter ICU stay $(61.89 \mu \mathrm{mol} / \mathrm{L}$, range $26.53-123.79 \mu \mathrm{mol} / \mathrm{L} ; 0.7 \mathrm{mg} /$ $\mathrm{dL}$, range $0.3-1.4 \mathrm{mg} / \mathrm{dL} ; \mathrm{z}=-2.3, \mathrm{p}=0.02$, Table 5). No other differences were seen in baseline characteristics in ICU LOS groups. Participants remaining in the ICU for longer periods demonstrated a significantly higher median $[\mathrm{Cr}]_{\max } \quad(106.1 \mu \mathrm{mol} / \mathrm{L}$, range $35.37-557.05 \mu \mathrm{mol} / \mathrm{L}$; $1.2 \mathrm{mg} / \mathrm{dL}$, range $0.4-6.3 \mathrm{mg} / \mathrm{dL}$ ) compared to those in the ICU for fewer than two days $(70.74 \mu \mathrm{mol} / \mathrm{L}$, range $35.37-194.52 \mu \mathrm{mol} / \mathrm{L} ; 0.8 \mathrm{mg} / \mathrm{dL}$, range $0.4-2.2 \mathrm{mg} / \mathrm{dL}$; $\mathrm{z}=-2.4, \mathrm{p}=0.02$, Table 5). Additionally, patients hospitalized for two or more days had a significantly higher $\left[\mathrm{Cl}^{-}\right]_{\max }$ (121.8 $\mathrm{mmol} / \mathrm{L}$, range $110.2-139.5 \mathrm{mmol} / \mathrm{L})$ compared to those with shorter ICU stay $(117.1 \mathrm{mmol} / \mathrm{L}$, range $109-127.1 \mathrm{mmol} / \mathrm{L} ; \mathrm{t}=-3.3, \mathrm{p}=0.002$; Table 5, Figure 3)

A total of 52 participants survived to hospital discharge. Among the baseline characteristics analyzed, only APPLE $_{\text {fast }}$ scores were different between mortality groups with those that died demonstrating a significantly higher score than those that survived (24.5, range 12-28 vs 19.5 , range $1-37 ; \mathrm{z}=-2.4, \mathrm{p}=0.02$, Table 6). Maximum median creatinine and corrected chloride concentrations did not differ between survivors and non-survivors (Table 6).

Across the entire study population, the median total IV fluid volume administered was $950 \mathrm{~mL}$ (range 120-14,400 mL), the median total IV fluid volume administered per kilogram was $111 \mathrm{~mL} / \mathrm{kg}$ (range
Table 4 Demographic and Patient Clinical Data Classified by Development of Hyperchloremia. Values are Presented as Mean and Standard Deviation for Normally Distributed Data and Median with Range for Non-Normally Distributed Data

\begin{tabular}{|c|c|c|c|}
\hline \multirow[t]{3}{*}{ Characteristics } & \multicolumn{2}{|l|}{ Hyperchloremia } & \multirow[t]{3}{*}{ p-value } \\
\hline & Yes & No & \\
\hline & $n=6$ & $n=54$ & \\
\hline Age (years) & $9.75(5-14.5)$ & $7.75(0.67-15.5)$ & 0.29 \\
\hline Weight (kg) & $8.5(2.0-29.0)$ & $9.75(2.64-43)$ & 0.68 \\
\hline $\begin{array}{l}\text { Heart rate } \\
\text { (beats } / \mathrm{min} \text { ) }\end{array}$ & $137 \pm 34$ & $140 \pm 34$ & 0.87 \\
\hline Temperature $\left({ }^{\circ} \mathrm{C}\right)$ & $38 \pm 1.1$ & $38.5 \pm 1.2$ & 0.33 \\
\hline $\mathrm{SBP}(\mathrm{mmHg})$ & $168 \pm 30.2(n=3)$ & $\begin{array}{l}138.4 \pm 35.2 \\
(n=32)\end{array}$ & 0.17 \\
\hline $\operatorname{MAP}(\mathrm{mmHg})$ & $123.5 \pm 33.2(n=2)$ & $\begin{array}{l}113.1 \pm 32.1 \\
(n=23)\end{array}$ & 0.69 \\
\hline APPLEfast score & $20(3-25)$ & $20(1-37)$ & 0.52 \\
\hline $\begin{array}{l}{\left[\mathrm{Cl}_{\text {corr }}\right] \text { on }} \\
\text { presentation } \\
(\mathrm{mmol} / \mathrm{L})\end{array}$ & $113.0 \pm 6.74$ & $109.7 \pm 5.58$ & 0.18 \\
\hline$\left[\mathrm{Cl}_{\mathrm{corr}}\right]_{\max }(\mathrm{mmol} / \mathrm{L})$ & $127.45(126.5-139.5)$ & $118.5(109-125.8)$ & $* * 0.0001$ \\
\hline $\begin{array}{l}{[\mathrm{Cr}] \text { on presentation }} \\
(\mu \mathrm{mol} / \mathrm{L}) \\
(\mathrm{mg} / \mathrm{dL})\end{array}$ & $\begin{array}{l}57.47(35.37-433.26) \\
0.65(0.4-4.9)\end{array}$ & $\begin{array}{l}70.74 \\
(26.53-2 \mid 2.21) \\
0.8(0.3-2.4)\end{array}$ & 0.74 \\
\hline $\begin{array}{l}{[\mathrm{Cr}]_{\max }(\mu \mathrm{mol} / \mathrm{L})} \\
(\mathrm{mg} / \mathrm{dL})\end{array}$ & $\begin{array}{l}110.53 \\
(61.89-557.05) \\
1.25(0.7-6.3)\end{array}$ & $\begin{array}{l}75.16 \\
(35.37-221.05) \\
0.85(0.4-2.5)\end{array}$ & 0.16 \\
\hline
\end{tabular}

Note: **Denotes $\mathrm{p}$-value of significance $(\mathrm{p}<0.05)$.

Abbreviations: SBP, systolic blood pressure; MAP, mean arterial pressure; [ $\left.\mathrm{Cl}_{\mathrm{corr}}\right]$, corrected chloride concentration; [Cr], creatinine concentration; $[\mathrm{Cr}]_{\max }$, maximum creatinine concentration; $\left[\mathrm{Cl}_{\text {corr }}\right]_{\max }$, maximum corrected chloride concentration.

21-334.8 $\mathrm{mL} / \mathrm{kg}$ ), the median total chloride delivered was $106.6 \mathrm{mmol}$ (range 19.5-1718.04 mmol), and the median VACL was $0.11 \mathrm{mmol} / \mathrm{L}(0.057-0.21 \mathrm{mmol} / \mathrm{L})$. The volumes of total fluid and crystalloid fluid delivered, and the total amount of chloride and VACL for each outcome group are outlined in Tables 7-10. Total crystalloid volume administered per kilogram was significantly higher in the hyperchloremic group $(143.2 \mathrm{~mL} / \mathrm{kg}$, range 88.1-243 $\mathrm{mL} / \mathrm{kg}$ ) vs the non-hyperchloremic group (94.1 mL/kg, range 18.6-242.4 mL/kg; $\mathrm{p}=0.04$, Table 7). Following adjustment for volume of crystalloid administered per kilogram per day, this difference was no longer significant $(\mathrm{p}=0.61$, Table 7$)$. There was no significant difference in the total volume of IV fluid administered between groups, with the exception of the ICU length of stay group $(\mathrm{p}=0.0005)$ (Table 8$)$. The total chloride amount delivered was significantly different between LOS $\geq 2$ days $(211.1 \mathrm{mmol}$, range $31.8-1718 \mathrm{mmol})$ and LOS $<2$ days $(66 \mathrm{mmol}$, range $19.5-384.3)(\mathrm{p}=0.0006$, Table 8$)$. 
Table 5 Demographic and Patient Clinical Data Classified by ICU Length of Stay. Values are Presented as Mean and Standard Deviation for Normally Distributed Data and Median with Range for Non-Normally Distributed Data

\begin{tabular}{|c|c|c|c|}
\hline \multirow[t]{3}{*}{ Characteristics } & \multicolumn{2}{|c|}{ ICU Length of Stay } & \multirow[t]{3}{*}{ p-value } \\
\hline & $\geq 2$ days & $<2$ days & \\
\hline & $n=26$ & $n=34$ & \\
\hline Age (years) & $8.5(I-14.5)$ & $7(0.67-15.5)$ & 0.66 \\
\hline Weight (kg) & $9.5(2.0-43)$ & $9.9(2.64-40)$ & 0.69 \\
\hline $\begin{array}{l}\text { Heart rate (beats/ } \\
\min \text { ) }\end{array}$ & $135 \pm 34$ & $143 \pm 33$ & 0.36 \\
\hline $\begin{array}{l}\text { Mean temperature } \\
\left({ }^{\circ} \mathrm{C}\right)\end{array}$ & $38.4 \pm 1.3$ & $38.5 \pm 1.1$ & 0.91 \\
\hline $\mathrm{SBP}(\mathrm{mmHg})$ & $\begin{array}{l}\mid 27(70-|8|) \\
(n=\mid 6)\end{array}$ & $\begin{array}{l}142(90-205) \\
(n=19)\end{array}$ & $* * 0.02$ \\
\hline MAP $(\mathrm{mmHg})$ & $\begin{array}{l}89.5(67-160) \\
(n=12)\end{array}$ & $\begin{array}{l}120(86-174) \\
(n=13)\end{array}$ & $* * 0.01$ \\
\hline APPLEfast score & $20(I-34)$ & $19.5(11-37)$ & 0.89 \\
\hline $\begin{array}{l}{\left[\mathrm{Cl}_{\text {corr }}\right] \text { on }} \\
\text { presentation } \\
(\mathrm{mmol} / \mathrm{L})\end{array}$ & $110.9 \pm 7.45$ & $109.4 \pm 3.95$ & 0.30 \\
\hline$\left[\mathrm{Cl}_{\text {corr }}\right]_{\max }(\mathrm{mmol} / \mathrm{L})$ & $|2| .8 \pm 5.93$ & $117.5 \pm 4.25$ & $* * 0.002$ \\
\hline $\begin{array}{l}{[\mathrm{Cr}] \text { on presentation }} \\
(\mu \mathrm{mol} / \mathrm{L}) \\
(\mathrm{mg} / \mathrm{dL})\end{array}$ & $\begin{array}{l}88.42 \\
(35.37-433.26) \\
1.0(0.4-4.9)\end{array}$ & $\begin{array}{l}61.89 \\
(26.53-123.79) \\
0.7(0.3-1.4)\end{array}$ & $* * 0.02$ \\
\hline $\begin{array}{l}{[\mathrm{Cr}]_{\max }(\mu \mathrm{mol} / \mathrm{L})} \\
(\mathrm{mg} / \mathrm{dL})\end{array}$ & $\begin{array}{l}\text { I06.I } \\
(35.37-557.05) \\
\text { I.2(0.4-6.3) }\end{array}$ & $\begin{array}{l}70.74 \\
(35.37-194.52) \\
0.8(0.4-2.2)\end{array}$ & $* * 0.02$ \\
\hline
\end{tabular}

Note: **Denotes $p$-value of significance $(p<0.05)$.

Abbreviations: SBP, systolic blood pressure; MAP, mean arterial pressure; [ $\mathrm{Cl}_{\text {corr }}$ ], corrected chloride concentration; $[\mathrm{Cr}]$, creatinine concentration; $[\mathrm{Cr}]_{\max }$, maximum creatinine concentration; $\left[\mathrm{Cl}_{\text {corr }}\right]_{\max }$, maximum corrected chloride concentration.

Volume-adjusted chloride load (VACL) was not significantly different in the AKI group (median $0.11 \mathrm{mmol} / \mathrm{L}$, range $0.06-0.16 \mathrm{mmol} / \mathrm{L}$ ) compared to the non-AKI group (median $0.11 \mathrm{mmol} / \mathrm{L}$, range $0.09-0.21 \mathrm{mmol} / \mathrm{L} ; \mathrm{z}=0.896$, $\mathrm{p}=0.3$, Table 9, Figure 4). After normalizing VACL by baseline body weight, the median VACL/kg was still not significantly different between those in the non-AKI group $(0.01 \mathrm{mmol} / \mathrm{L} / \mathrm{kg}$, range $0.003-0.08 \mathrm{mmol} / \mathrm{L} / \mathrm{kg})$ and those in the AKI group $(0.02 \mathrm{mmol} / \mathrm{L} / \mathrm{kg}$, range $0.002-0.03$ $\mathrm{mmol} / \mathrm{L} / \mathrm{kg})(\mathrm{z}=1.53, \mathrm{p}=0.13$, Table 9$)$. Volume-adjusted chloride load (VACL) was not significantly different between any other group under comparison. There was no significant difference in the volume of IV crystalloid fluid administered between the AKI (median $59.5 \mathrm{~mL} / \mathrm{kg}$ / day, range $18.6-95.3 \mathrm{~mL} / \mathrm{kg} /$ day) and non-AKI groups $(56.7 \mathrm{~mL} / \mathrm{kg} /$ day, range $14.1-123.0 \mathrm{~mL} / \mathrm{kg} /$ day; $\mathrm{p}=0.88$, Table 9). There was no significant difference in total

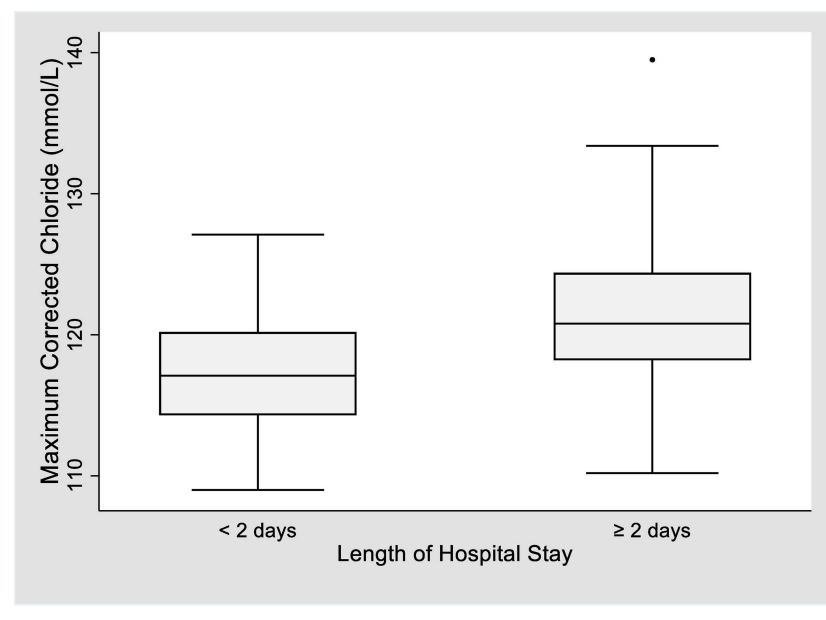

Figure 3 Box and whisker plot of maximum corrected chloride concentration between length of stay groups.

Notes: Boxes represent the interquartile range from the 25 th to the 75 th percentile. The horizontal bar in each box represents the median value. The T-bars represent the range. The black dots represent outliers. The reference interval for chloride is $106-126 \mathrm{mmol} / \mathrm{L}$. The median maximum corrected chloride concentration was significantly higher in LOS $\geq 2$ days group ( $120.8 \mathrm{mmol} / \mathrm{L}$; range 1 I $0-139.5$ $\mathrm{mmol} / \mathrm{L}$ ) as compared to the LOS < 2 days group ( $117.1 \mathrm{mmol} / \mathrm{L}$; range $109-127.1$ $\mathrm{mmol} / \mathrm{L})(p=0.002)$.

volume of IV fluid volume administered, crystalloid fluid volume administered, median total chloride delivered or VACL between mortality groups (Table 10).

\section{Discussion}

Maximum corrected chloride concentration $\left([\mathrm{Cl}]_{\max }\right)$ was significantly higher in dogs with hospital-acquired AKI versus those without. Additionally, $[\mathrm{Cl}]_{\max }$ was significantly higher in dogs hospitalized in the ICU 2 days or longer vs those hospitalized for fewer than 2 days. These findings are similar to the results of several human studies investigating the effect of chloride concentration on morbidity and mortality. In people, numerous studies have shown that changes in serum chloride concentration, independent of serum sodium and bicarbonate, are associated with increased risk of AKI. ${ }^{14-16}$ Hyperchloremia has also been shown to be independently associated with increased ICU and hospital length of stay. ${ }^{17,18}$

As the major anion filtered by the glomeruli and reabsorbed in the renal tubules, chloride functions in the retention and secretion fluid both directly from intravascular and extravascular compartments, and indirectly through tubuloglomerular feedback. ${ }^{19,20}$ Renal blood supply is significantly affected by fluctuations in serum chloride concentration. In addition to the influence of chloride on renal autoregulation, several mechanisms of the injurious effects of chloride dysregulation have been proposed 
Table 6 Demographic and Patient Clinical Data Classified by Mortality. Values are Presented as Mean and Standard Deviation for Normally Distributed Data and Median with Range for NonNormally Distributed Data

\begin{tabular}{|c|c|c|c|}
\hline \multirow[t]{3}{*}{ Characteristics } & \multicolumn{2}{|l|}{ Mortality } & \multirow[t]{3}{*}{ p-value } \\
\hline & Death & Survival & \\
\hline & $n=8$ & $n=52$ & \\
\hline Age (years) & $8.5(3.5-15.5)$ & $7.25(0.67-15)$ & 0.23 \\
\hline Weight (kg) & $19.65(3.8-4.3)$ & $8.95(2-42.2)$ & 0.09 \\
\hline $\begin{array}{l}\text { Heart rate } \\
\text { (beats/min) }\end{array}$ & $153 \pm 37$ & $137 \pm 33$ & 0.22 \\
\hline Temperature $\left({ }^{\circ} \mathrm{C}\right)$ & $38.7 \pm 0.9$ & $38.4 \pm 1.2$ & 0.58 \\
\hline SBP $(\mathrm{mmHg})$ & $130 \pm 43(n=6)$ & $\begin{array}{l}143 \pm 34 \\
(n=29)\end{array}$ & 0.42 \\
\hline MAP (mmHg) & $\begin{array}{l}67(67-67) \\
(n=1)\end{array}$ & $\begin{array}{l}109(73-174) \\
(n=24)\end{array}$ & 0.1 \\
\hline APPLEfast score & $24.5(12-28)$ & $19.5(I-37)$ & $* * 0.02$ \\
\hline $\begin{array}{l}{\left[\mathrm{Cl}_{\text {corr }}\right] \text { on }} \\
\text { presentation } \\
(\mathrm{mmol} / \mathrm{L})\end{array}$ & $108.7 \pm 2.79$ & $110.2 \pm 6.05$ & 0.49 \\
\hline$\left[\mathrm{Cl}_{\mathrm{corr}}\right]_{\max }(\mathrm{mmol} / \mathrm{L})$ & $\begin{array}{l}120.8 \\
(I 11.1-127.1)\end{array}$ & $\begin{array}{l}118.7 \\
(109.0-139.5)\end{array}$ & 0.41 \\
\hline $\begin{array}{l}{[\mathrm{Cr}] \text { on presentation }} \\
(\mu \mathrm{mol} / \mathrm{L}) \\
(\mathrm{mg} / \mathrm{dL})\end{array}$ & $\begin{array}{l}48.63 \\
(35.37-132.63) \\
0.55(0.4-1.5)\end{array}$ & $\begin{array}{l}70.74 \\
(35.37-433.26) \\
0.8(0.4-4.9)\end{array}$ & 0.50 \\
\hline $\begin{array}{l}{[\mathrm{Cr}]_{\max }(\mu \mathrm{mol} / \mathrm{L})} \\
(\mathrm{mg} / \mathrm{dL})\end{array}$ & $\begin{array}{l}114.95 \\
(44.21-194.52) \\
1.3(0.5-2.2)\end{array}$ & $\begin{array}{l}70.74 \\
(35.37-557.05) \\
0.8(0.4-6.3)\end{array}$ & 0.17 \\
\hline
\end{tabular}

Note: **Denotes $p$-value of significance $(p<0.05)$.

Abbreviations: SBP, systolic blood pressure; MAP, mean arterial pressure; [Cl $\mathrm{Clrr}_{\text {cr }}$, corrected chloride concentration; $[\mathrm{Cr}]$, creatinine concentration; $[\mathrm{Cr}]_{\max }$, maximum creatinine concentration; $\left[\mathrm{Cl}_{\text {corr }}\right]_{\max }$, maximum corrected chloride concentration.

including mediation of vascular smooth-muscle cell calcium-dependent contraction, impairment of renal vascular responsiveness to endogenous vasoconstrictors, and modification of plasma renin activity and systemic blood pressure. $^{19,21,22}$ Ultimately, increasing plasma concentrations of chloride and the development of hyperchloremic metabolic acidosis have been proposed to lead to renal vasoconstriction, diminished glomerular filtration rate, renal dysfunction, and decreased splanchnic perfusion, ${ }^{23}$ all of which have the potential to worsen outcomes in critically ill patients.

Metabolic acidosis is a frequently encountered acidbase disturbance in ill patients and is associated with both $\mathrm{AKI}^{24}$ and mortality. ${ }^{25-27}$ Hyperchloremia is an important contributor to normal anion-gap metabolic acidosis, and in many processes, including renal acidbase handling, intestinal bicarbonate secretion, gastric
Table 7 Total Fluid Volume Administered, Crystalloid Volume Administered, Median Total Chloride Delivered and Median VACL in Hyperchloremia Groups

\begin{tabular}{|c|c|c|c|}
\hline \multirow[t]{3}{*}{ Variables } & \multicolumn{2}{|c|}{ Hyperchloremia } & \multirow[t]{3}{*}{ p-value } \\
\hline & Yes & No & \\
\hline & $n=6$ & $n=54$ & \\
\hline Fluid $_{\text {tot }}(\mathrm{mL})$ & $\begin{array}{l}1300 \\
(550-4400)\end{array}$ & $\begin{array}{l}950 \\
(120-14,400)\end{array}$ & 0.46 \\
\hline Fluid $_{\text {tot }} / \mathrm{kg}(\mathrm{mL} / \mathrm{kg})$ & $\begin{array}{l}154.5 \\
(95.8-277.0)\end{array}$ & $\begin{array}{l}106.6 \\
(21.0-334.82)\end{array}$ & 0.06 \\
\hline $\begin{array}{l}\text { Crystalloid }_{\text {tot }} / \mathrm{kg} \\
(\mathrm{mL} / \mathrm{kg})\end{array}$ & $\begin{array}{l}143.2 \\
(88.1-243)\end{array}$ & $\begin{array}{l}94.1 \\
(18.6-242.4)\end{array}$ & $* * 0.04$ \\
\hline $\begin{array}{l}\text { Daily crystalloid }{ }_{\text {tot }} / \mathrm{kg} \\
(\mathrm{mL} / \mathrm{kg} / \text { day })\end{array}$ & $\begin{array}{l}69.1 \\
(29.4-94.6)\end{array}$ & $\begin{array}{l}56.6 \\
(14.1-123.0)\end{array}$ & 0.61 \\
\hline $\mathrm{Cl}_{\text {tot }}^{-}(\mathrm{mmol})$ & $\begin{array}{l}144.9 \\
(31.8-510.1)\end{array}$ & $\begin{array}{l}101.9 \\
(19.5-1718.0)\end{array}$ & 0.59 \\
\hline VACL (mmol/L) & $\begin{array}{l}0.11 \\
(0.06-0.11)\end{array}$ & $\begin{array}{l}0.11 \\
(0.06-0.21)\end{array}$ & 0.4 \\
\hline VACL/kg (mmol/L/kg) & $\begin{array}{l}0.01 \\
(0.004-0.03)\end{array}$ & $\begin{array}{l}0.01 \\
(0.002-0.08)\end{array}$ & 0.73 \\
\hline
\end{tabular}

Note: $* *$ Denotes $p$-values of significance $(p<0.05)$.

Abbreviations: Fluid ${ }_{\text {tot }}$, total fluid administered $(\mathrm{mL})$; Fluid ${ }_{\text {tot }} / \mathrm{kg}$, total fluid volume administered per kilogram; Crystalloid $d_{\text {tot }} / \mathrm{kg}$, total volume of crystalloid fluid administered per $\mathrm{kg}$; Daily crystalloid $\mathrm{tot}_{\mathrm{t}} / \mathrm{kg}$, total volume of crystalloid fluid administered per kilogram per day; $\mathrm{Cl}^{-}$tot, total chloride delivered (mmol); $\mathrm{VACL}$, volumeadjusted chloride load; VACL/kg, volume-adjusted chloride load adjusted by baseline body weight.

Table 8 Total Fluid Volume Administered, Crystalloid Volume Administered, Median Total Chloride Delivered and Median VACL in Length of Stay Groups

\begin{tabular}{|c|c|c|c|}
\hline \multirow[t]{3}{*}{ Variables } & \multicolumn{2}{|c|}{ Length of Stay } & \multirow[t]{3}{*}{ p-value } \\
\hline & $\geq 2$ days & $<2$ days & \\
\hline & $n=26$ & $n=34$ & \\
\hline Fluid $_{\text {tot }}(\mathrm{mL})$ & $\begin{array}{l}1820 \\
(400-14,400)\end{array}$ & $\begin{array}{l}620 \\
(120-4100)\end{array}$ & $* * 0.0005$ \\
\hline Fluid $_{\text {tot }} / \mathrm{kg}(\mathrm{mL} / \mathrm{kg})$ & $\begin{array}{l}169.4 \\
(95.8-334.8)\end{array}$ & $\begin{array}{l}67.1 \\
(21.0-150.1)\end{array}$ & $* * 0.0000 \mathrm{I}$ \\
\hline $\begin{array}{l}\text { Crystalloid } \text { tot } / \mathrm{kg}(\mathrm{mL} / \\
\mathrm{kg})\end{array}$ & $\begin{array}{l}154.6 \\
(88.1-243)\end{array}$ & $\begin{array}{l}56.0 \\
(18.6-142)\end{array}$ & $* * 0.00001$ \\
\hline $\begin{array}{l}\text { Daily crystalloid } \text { tot } / \mathrm{kg} \\
(\mathrm{mL} / \mathrm{kg} / \text { day })\end{array}$ & $\begin{array}{l}61.9 \\
(29.4-105.0)\end{array}$ & $\begin{array}{l}52.0 \\
(14.1-123.0)\end{array}$ & 0.1 \\
\hline $\mathrm{Cl}_{\text {tot }}^{-}(\mathrm{mmol})$ & $\begin{array}{l}211.1 \\
(31.8-1718.0)\end{array}$ & $\begin{array}{l}66.0 \\
(19.5-384.3)\end{array}$ & $* * 0.0006$ \\
\hline VACL (mmol/L) & $\begin{array}{l}0.11 \\
(0.06-0.15)\end{array}$ & $\begin{array}{l}0.11 \\
(0.09-0.21)\end{array}$ & 0.69 \\
\hline $\mathrm{VACL} / \mathrm{kg}(\mathrm{mmol} / \mathrm{L} / \mathrm{kg})$ & $\begin{array}{l}0.01 \\
(0.002-0.04)\end{array}$ & $\begin{array}{l}0.01 \\
(0.003-0.08)\end{array}$ & 0.67 \\
\hline
\end{tabular}

Note: **Denotes $p$-values of significance $(p<0.05)$.

Abbreviations: Fluid tor, total fluid administered $(\mathrm{mL})$; Fluid tot $/ \mathrm{kg}$, total fluid volume administered per kilogram; Crystalloid ${ }_{\text {tot }} / \mathrm{kg}$, total volume of crystalloid fluid administered per $\mathrm{kg}$; Daily crystalloid $\mathrm{too}_{\mathrm{t}} / \mathrm{kg}$, total volume of crystalloid fluid administered per kilogram per day; $\mathrm{Cl}^{-}$tot total chloride delivered (mmol); $\mathrm{VACL}$, volume-adjusted chloride load; $\mathrm{VACL} / \mathrm{kg}$, volume-adjusted chloride load adjusted by baseline body weight. 
Table 9 Total Fluid Volume Administered, Crystalloid Volume Administered, Median Total Chloride Delivered and Median VACL in AKI Groups

\begin{tabular}{|c|c|c|c|}
\hline \multirow[t]{3}{*}{ Variables } & \multicolumn{2}{|l|}{ AKI } & \multirow[t]{3}{*}{ p-value } \\
\hline & Yes & No & \\
\hline & $n=15$ & $n=45$ & \\
\hline Fluid $_{\text {tot }}(\mathrm{mL})$ & $\begin{array}{l}1760 \\
(160-9270)\end{array}$ & $\begin{array}{l}850 \\
(120-14,400)\end{array}$ & 0.06 \\
\hline Fluid $_{\text {tot }} / \mathrm{kg}(\mathrm{mL} / \mathrm{kg})$ & $\begin{array}{l}150.1 \\
(21.0-299.7)\end{array}$ & $\begin{array}{l}107.8 \\
(21.6-334.82)\end{array}$ & 0.14 \\
\hline $\begin{array}{l}\text { Crystalloid }{ }_{\text {tot }} / \mathrm{kg}(\mathrm{mL} / \\
\mathrm{kg})\end{array}$ & $\begin{array}{l}122.5 \\
(18.6-243)\end{array}$ & $\begin{array}{l}99.4 \\
(19.7-242.4)\end{array}$ & 0.22 \\
\hline $\begin{array}{l}\text { Daily crystalloid }{ }_{\text {tot }} / \mathrm{kg} \\
(\mathrm{mL} / \mathrm{kg} / \text { day })\end{array}$ & $\begin{array}{l}59.5 \\
(18.6-95.3)\end{array}$ & $\begin{array}{l}56.7 \\
(14.1-123.0)\end{array}$ & 0.88 \\
\hline $\mathrm{Cl}_{\text {tot }}^{-}(\mathrm{mmol})$ & $\begin{array}{l}192.9 \\
(19.5-934.7)\end{array}$ & $\begin{array}{l}88.0 \\
(21.6-1718.0)\end{array}$ & 0.1 \\
\hline $\mathrm{VACL}(\mathrm{mmol} / \mathrm{L})$ & $\begin{array}{l}0.11 \\
(0.06-0.17)\end{array}$ & $\begin{array}{l}0.11 \\
(0.09-0.21)\end{array}$ & 0.37 \\
\hline VACL/kg (mmol/L/kg) & $\begin{array}{l}0.02 \\
(0.002-0.03)\end{array}$ & $\begin{array}{l}0.01 \\
(0.003-0.08)\end{array}$ & 0.13 \\
\hline
\end{tabular}

Abbreviations: Fluid ${ }_{\text {tot }}$, total fluid administered $(\mathrm{mL})$; Fluid $_{\text {tot }} / \mathrm{kg}$, total fluid volume administered per kilogram; Crystalloid ${ }_{\text {tot }} / \mathrm{kg}$, total volume of crystalloid fluid administered per kg; Daily crystalloid ${ }_{\text {tot }} / \mathrm{kg}$, total volume of crystalloid fluid administered per kilogram per day; $\mathrm{Cl}^{-}$tot, total chloride delivered (mmol); $\mathrm{VACL}$, volumeadjusted chloride load; VACL/kg, volume-adjusted chloride load adjusted by baseline body weight.

Table 10 Total Fluid Volume Administered, Crystalloid Volume Administered, Median Total Chloride Delivered and Median VACL in Mortality Groups

\begin{tabular}{|c|c|c|c|}
\hline \multirow[t]{3}{*}{ Variables } & \multicolumn{2}{|l|}{ Mortality } & \multirow[t]{3}{*}{ p-value } \\
\hline & Death & Survival & \\
\hline & $n=8$ & $n=52$ & \\
\hline Fluid $_{\text {tot }}(\mathrm{mL})$ & $\begin{array}{l}1900 \\
(210-14,400)\end{array}$ & $\begin{array}{l}850 \\
(120-9270)\end{array}$ & 0.07 \\
\hline Fluid $_{\text {tot }} / \mathrm{kg}(\mathrm{mL} / \mathrm{kg})$ & $\begin{array}{l}\text { II } 8.3 \\
(55.6-334.82)\end{array}$ & $\begin{array}{l}I I I \\
(21.1-3 \mid 3.0)\end{array}$ & 0.41 \\
\hline Crystalloid $_{\text {tot }} / \mathrm{kg}(\mathrm{mL} / \mathrm{kg})$ & $\begin{array}{l}107.8 \\
(41.1-242.4)\end{array}$ & $\begin{array}{l}100.3 \\
(18.6-243)\end{array}$ & 0.62 \\
\hline $\begin{array}{l}\text { Daily crystalloid } \text { tot } / \mathrm{kg} \\
(\mathrm{mL} / \mathrm{kg} / \text { day })\end{array}$ & $\begin{array}{l}54.1 \\
(41.0-94.6)\end{array}$ & $\begin{array}{l}58.4 \\
(14.1-123.0)\end{array}$ & 0.72 \\
\hline $\mathrm{Cl}_{\text {tot }}^{-}(\mathrm{mmol})$ & $\begin{array}{l}211.5 \\
(21.7-|7| 8.0)\end{array}$ & $\begin{array}{l}85.8 \\
(19.5-934.7)\end{array}$ & 0.06 \\
\hline VACL (mmol/L) & $\begin{array}{l}0.11 \\
(0.09-0.16)\end{array}$ & $\begin{array}{l}0.11 \\
(0.06-0.21)\end{array}$ & 0.9 \\
\hline $\mathrm{VACL} / \mathrm{kg}(\mathrm{mmol} / \mathrm{L} / \mathrm{kg})$ & $\begin{array}{l}0.007 \\
(0.002-0.03)\end{array}$ & $\begin{array}{l}0.01 \\
(0.002-0.08)\end{array}$ & 0.1 \\
\hline
\end{tabular}

Abbreviations: Fluid tot , total fluid administered $(\mathrm{mL})$; Fluid ${ }_{\text {tot }} / \mathrm{kg}$, total fluid volume administered per kilogram; Crystalloid tot $/ \mathrm{kg}$, total volume of crystalloid fluid administered per kg; Daily Crystalloid ${ }_{\text {tot }} / \mathrm{kg}$, total volume of crystalloid fluid administered per kilogram per day; $\mathrm{Cl}^{-}$tot, total chloride delivered (mmol); $\mathrm{VACL}$, volumeadjusted chloride load; $\mathrm{VACL} / \mathrm{kg}$, volume-adjusted chloride load adjusted by baseline body weight.

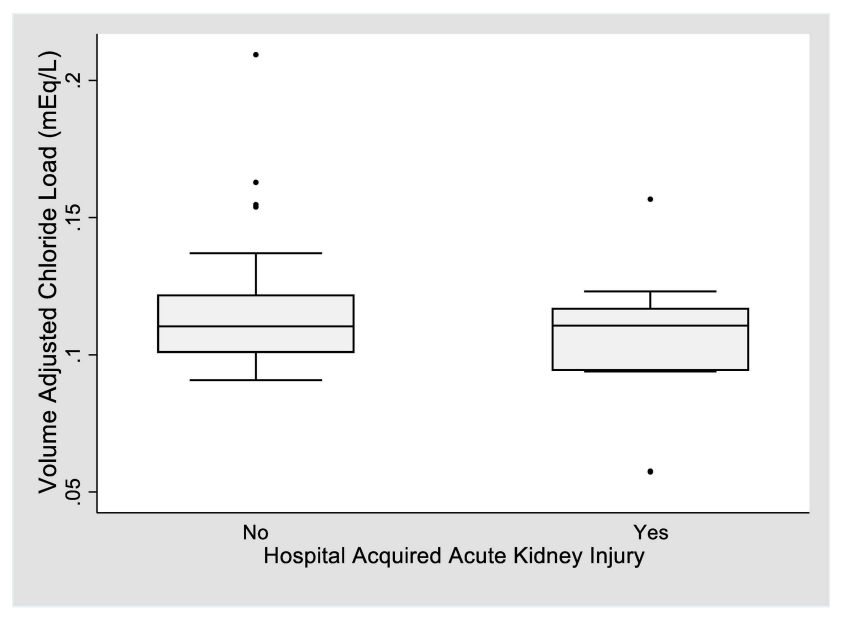

Figure 4 Box and whisker plot of volume-adjusted chloride load between AKI groups. Notes: Boxes represent the interquartile range from the 25 th to the 75 th percentile. The horizontal bar in each box represents the median value. The T-bars represent the range. The black dots represent outliers. There was no significant difference in the median $V A C L$ in the AKI group $(0.11 \mathrm{mmol} / \mathrm{L}$; range $0.06-0.17$ $\mathrm{mmol} / \mathrm{L})$ and non-AKI group $(0.11 \mathrm{mmol} / \mathrm{L}$; range $0.09-0.21 \mathrm{mmol} / \mathrm{L})(p=0.3)$.

acid secretion and transcellular ion exchange, chloride and bicarbonate are reciprocally linked. ${ }^{28}$ Perioperative hyperchloremic metabolic acidosis has been shown to be associated with AKI. ${ }^{16,29}$ Several studies have shown that high serum chloride concentrations are associated with outcome, including AKI and mortality independent of serum bicarbonate concentration. ${ }^{14,30}$ Interestingly, the type of metabolic acidosis appears to be more highly predictive of adverse outcomes than the severity of acidosis, with poorer outcomes associated with unmeasured anion accumulation and lactic acidosis. ${ }^{31,32}$ As this study did not aim to investigate relationships between chloride and metabolic acidosis, or the effect of hyperchloremic metabolic acidosis on outcome, bicarbonate concentration was not available for analysis and inclusion into collected data. Future investigations regarding the effect of chloride on AKI and mortality may benefit from concurrent quantitative and qualitative acid-base analysis to determine the independent effects of maximum and change in chloride concentration.

In this study, $[\mathrm{Cl}]_{\max }$ was not significantly different in mortality groups. This finding stands in contrast to the results of some human studies, which have found that patients who were either hyperchloremic or who had within-subject worsening of hyperchloremia had increased mortality. ${ }^{15,33,34}$ Many of the studies investigating the impact of chloride on mortality were large enough to perform multivariate analysis to adjust for illness severity and found independent associations between hyperchloremia 
or worsened hyperchloremia and mortality. ${ }^{15,33,34}$ In the current study, there was a significant difference in APPLE $_{\text {fast }}$ score on presentation between survivors and non-survivors, however, with only 8 patients in the nonsurvivor group, we are not able to adjust for illness severity to determine an independent association between chloride and mortality.

Systolic blood pressure on presentation was significantly higher in dogs that developed AKI while the mean arterial pressure was not significantly different between groups. The elevated systolic blood pressure in patients with AKI may be due to previously undiagnosed hypertension, chronic hypertension, or may signify compensated shock, all of which can lead to acute kidney injury. ${ }^{35-38}$ Conversely, the baseline systolic and mean blood pressures were significantly lower in patients hospitalized for 2 days or longer, regardless of the development of AKI. The median blood pressures of patients in the longer hospitalization group were within normal limits on presentation; however, those with shorter ICU stay were on average hypertensive on presentation.

The significance of abnormal systolic or mean arterial pressure on morbidity and mortality is well studied in human medicine; however, much of the work has been related to the effect of blood pressure on cardiovascular outcome or performed in patients with known or suspected cardiovascular disease. $^{39-42}$ Patients with chronic hypertension, or patients with underlying cardiovascular disease experience alterations in their hemodynamic autoregulatory curve, whereby lower blood pressures are deleterious to organ perfusion. ${ }^{43,44}$ In this study, patients with known pre-existing hypertension, $\mathrm{CKD}$ or patients receiving antihypertensive medication were excluded; however, a number of other conditions which were not exclusion criteria, including occult or early hypertension, underlying endocrinopathy, cardiac or hepatic disease, may have contributed to alterations in blood pressure. Additionally, pain-, anxiety- or excitement-induced situational hypertension, likely impacted blood pressure results. ${ }^{45}$

Baseline creatinine and maximum creatinine were also significantly higher in patients hospitalized for $\geq 2$ days. This may be due to the inclusion of patients with AKI on presentation to the emergency room, or may be reflective of underlying disease severity, even though APPLE $_{\text {fast }}$ scores at the time of emergency room presentation did not differ between the two length of stay populations. Additionally, it cannot be determined whether this finding is simply correlation or causation, ie, these patients may have had prolonged hospitalization due to elevations in creatinine.

Total fluid volume, total crystalloid volume, and total fluid volume adjusted for body weight were significantly different in length of stay groups. However, when adjusted for duration of hospitalization, there was no significant difference in crystalloid volume per day between groups. Additionally, while total chloride administered was also significantly different between length of stay groups, when normalized for volume of fluid administered, VACL showed no difference between length of stay groups. These findings likely reflect that duration of hospitalization influences the overall volume of fluids received as well as the volume of fluids of higher chloride content. Though the majority of patients received crystalloids with a sub-physiologic chloride concentration (PlasmaLyte-A chloride concentration 98 $\mathrm{mmol} / \mathrm{L}$; LRS chloride concentration $104 \mathrm{mmol} / \mathrm{L}$ ), hyperchloremia may develop due to a variety of endogenous factors including increased aldosterone level, proximal or distal tubular acidosis or compensation for respiratory alkalosis. $^{46}$ Additionally, aggressive potassium chloride supplementation is a source of exogenous chloride delivery which could contribute to observed hyperchloremia.

In humans, several widely publicized studies investigating the effect of chloride composition of intravenous fluids have demonstrated adverse effects associated with administration of chloride-rich fluids (eg, NS).$^{5,17,47-49}$ No dog in this study received normal saline as a primary crystalloid IV fluid, as this institution has an overwhelming preference towards using balanced crystalloids (specifically PlasmaLyte A and lactated Ringer's solution); however, ten percent of dogs developed elevations in corrected chloride concentration despite the use of IV fluid solutions with physiologic chloride concentrations. The exclusive use of balanced crystalloid solutions in this study likely led to similar VACL between all outcome groups. Future investigations with a wider range of electrolyte solutions would be necessary to further characterize the consequence of administration of large volumes of solutions containing supraphysiologic chloride concentrations.

The worsened outcomes with elevations in chloride concentration found in this study should be interpreted with caution, particularly alongside the consideration of well-described deleterious effects of excessive volume administration. ${ }^{50-54}$ Contrary to the human study which observed an association between VACL and mortality, ${ }^{8}$ the current prospective study did not observe an association between VACL and mortality, nor between VACL and 
AKI. This might suggest that the total volume of IV fluids administered in this study contributed to the development of AKI, rather than the administered chloride load itself. The injurious effects of greater total volume of fluid infused and positive fluid balance in critically ill human patients are well described in the literature..$^{50,55,56}$ Excessive volume administration leads to interstitial and organ edema, ${ }^{51}$ and renal impairment can occur subsequent to increased interstitial pressures or a consequence of abdominal compartment syndrome. Release of atrial natriuretic peptide secondary to hypervolemia increases renal salt and water excretion, reduces plasma volume and directly damages the endothelial glycocalyx. ${ }^{51}$ Positive fluid balance has been shown to be independently associated with both AKI and higher mortality rates. ${ }^{50,57}$ In the current study, there was no significant difference in the volume of IV fluid administered per kilogram nor the volume of crystalloid administered per day between AKI groups. Similarly, no differences were found between survival or hyperchloremia groups in either of these variables. Studies of larger populations of critically ill dogs may be necessary to better evaluate the effect of fluid administration volume and morbidity or mortality.

There are several considerable limitations to this study. The observational design limits the ability to assess causality and limits the ability to take cofounders into consideration when doing statistical analyses. The open-label, non-randomized design of this study may have confounded certain study outcome measures, such as length of stay, and may have impacted clinical decision-making regarding fluid administration; however, no significant difference in VACL was found in any of the outcome groups, nor was there significant difference in total crystalloid volume administered in $\mathrm{mL} / \mathrm{kg} /$ day. The small sample size of this observational study limited the ability to perform multivariate analysis. The overall power for evaluation of the effect of high corrected chloride or high VACL on survival is poor. With only eight dogs in the nonsurvivor group, it is possible that a difference in mortality would be detected with a larger sample size. Additionally, given the relatively low acuity of this dog population (as

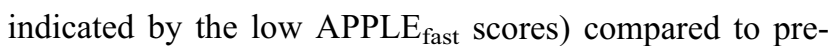
vious publications evaluating sicker patient cohorts $^{58}$ this study likely does not represent the general population of critically ill dogs. A similar study in dogs presenting with higher APPLE $_{\text {fast }}$ scores is indicated to determine the influence of chloride administration in a population with relatively greater illness severity.
In this study, maximum corrected chloride concentration and prevalence of hyperchloremia were significantly higher in dogs which developed hospital-acquired AKI. Maximum corrected chloride and creatinine concentrations were significantly different in patients with a length of stay greater than or equal to two days. This study failed to demonstrate a significant difference in volume-adjusted chloride load between AKI or mortality groups.

This study does demonstrate that hospitalized dogs with relatively low acuity are susceptible to developing acute kidney injury. Increased corrected chloride concentrations, even concentrations within the reference interval, are associated with acute kidney injury as defined by increase in creatinine concentration. Although this study was not designed to evaluate the pathophysiology underlying hyperchloremia and in-hospital AKI, careful trending of changes in chloride concentration may mitigate progression of acute kidney injury in hospitalized dogs. Dogs with underlying CKD, hypertension, cardiac disease, and/or sepsis are susceptible to acute kidney injury and may also benefit from trending of chloride concentration and avoidance of excessive chloride administration. Future analyses in populations receiving a wider range of chloride volumes, a wider range of illness acuity and a large sample size would be needed to better identify independent association of chloride on outcomes in critically ill dogs.

\section{Ethics Statement}

This study was performed at the Animal Medical Center in New York, USA. The study protocol was approved by the Animal Medical Center's Institutional Animal Care and Use Committee and informed client consent was obtained prior to study enrollment. This study adhered to veterinary care best practice guidelines.

\section{Acknowledgments}

From the Department of Emergency and Critical Care, The Animal Medical Center, New York.

\section{Disclosure}

Funding support provided by the Caspary Research Institute, The Animal Medical Center, New York. No additional grant support was used for this study. All work for this project was performed at the Animal Medical Center, New York. The authors declare that they have no conflict of interest with the contents of this article. 


\section{References}

1. Zager E, Fletcher DJ, Goggs R. Evaluation of the association between strong ion acid-base disturbances and mortality in dogs: a retrospective study. Vet Med Sci. 2018;4(3):206-217. doi:10.1002/ vms3.101

2. Boag AK, Coe RJ, Martinez TA, et al. Acid-base and electrolyte abnormalities in dogs with gastrointestinal foreign bodies. $J$ Vet Intern Med. 2005;19(16):816-821. doi:10.1111/j.1939-1676.2005.tb02770.x

3. Ueda Y, Hopper K, Epstein S. Incidence and prognosis associated with hyperchloremia and hypochloremia in dogs and cats. $J$ Vet Emerg Crit Care. 2016;26(Suppl 1):S10-11.

4. Goggs R, De Rosa S, Fletcher DJ. Electrolyte disturbances are associated with non-survival in dogs - a multivariable analysis. Front Vet Sci. 2017;4:135. doi:10.3389/fvets.2017.00135

5. Yunos NM, Bellomo R, Hegarty C, et al. Association between a chloride-liberal vs chloride-restrictive intravenous fluid administration strategy and kidney injury in critically ill adults. JAMA. 2012;308(15):1566-1572. doi:10.1001/jama.2012.13356

6. Young P, Bailey M, Beasley R, et al. Effect of a buffered crystalloid solution vs saline on acute kidney injury among patients in the intensive care unit: the SPLIT randomized clinical trial. JAMA. 2015;314(16):1701-1710. doi:10.1001/jama.2015.12334

7. Young PJ, Joannidis M. Crystalloid fluid therapy: is the balance tipping towards balanced solutions? Intensive Care Med. 2014;40 (12):1966-1968. doi:10.1007/s00134-014-3531-1

8. Shaw AD, Raghunathan K, Peyerl FW, et al. Association between intravenous chloride load during resuscitation and in-hospital mortality among patients with SIRS. Intensive Care Med. 2014;40 (12):1897-1905. doi:10.1007/s00134-014-3505-3

9. Hanson K, Linklater A. Placing a central sampling catheter through a peripheral vein. Clin Brief. 2014;57-62.

10. DiBartola SP, Bateman S. Introduction to fluid therapy. In: DiBartola SP, editor. Fluid, Electrolyte, and Acid Base Disorders in Small Animal Practice. 4th ed. St. Louis: Saunders; 2012:331-349.

11. Thoen ME, Kerl ME. Characterization of acute kidney injury in hospitalized dogs and evaluation of a veterinary acute kidney injury staging system. J Vet Emerg Crit Care. 2011;21(6):648-657.

12. Hayes G, Mathews K, Doig G, et al. The acute patient physiologic and laboratory evaluation (APPLE) score: a severity of illness stratification system for hospitalized dogs. J Vet Intern Med. 2010;24 (5):1034-1047. doi:10.1111/j.1939-1676.2010.0552.x

13. Babyak JM, Weiner DE, Noubary F, et al. Prevalence of elevated serum creatinine concentration in dogs presenting to a veterinary academic medical center (2010-2014). J Vet Intern Med. 2017;31 (6):1757-1764. doi:10.1111/jvim.14823

14. Suetrong B, Pisitsak C, Boyd JH, et al. Hyperchloremia and moderate increase in serum chloride are associated with AKI in severe sepsis and septic shock patients. Crit Care. 2016;20(1):315. doi:10.1186/ s13054-016-1499-7

15. Sen A, Keener CM, Sileanu FE, et al. Chloride content of fluids used for large volume resuscitation is associated with reduced survival. Crit Care. 2017;45(2):146-153. doi:10.1097/CCM.0000000000002063

16. Oh TK, Kim CY, Jeon YT, et al. Perioperative hyperchloremia and its association with postoperative acute kidney injury after craniotomy for primary brain tumor resection: a retrospective, observational study. J Neurosurg Anesthesiol. 2019;31(3):311-317. doi:10.1097/ ANA.0000000000000512

17. Shaw AD, Schermer CR, Lobo DN, et al. Impact of intravenous fluid composition on outcomes in patients with systemic inflammatory response syndrome. Crit Care. 2015;19(1):334. doi:10.1186/s13054015-1045-z

18. McCluskey SA, Karkouti K, Wijeysundera D, et al. Hyperchloremia after noncardiac surgery is independently associated with increased morbidity and mortality: a propensity-matched cohort study. Anesth Analg. 2013;117(2):412-421. doi:10.1213/ANE.0b013e318293d81e
19. Wright FS, Briggs P. Feedback regulation of glomerular filtration rate. Am J Physiol. 1977;233(1):F1-F7.

20. Wilcox CS. Regulation of renal blood flow by plasma chloride. $J$ Clin Invest. 1983;71(3):726-735. doi:10.1172/JCI110820

21. Kotchen TA, Luke RG, Ott CE, et al. Effect of chloride on renin and blood pressure responses to sodium chloride. Ann Intern Med. 1983;98(5):817-822. doi:10.7326/0003-4819-98-5-817

22. Hansen PB, Jensen BL, Skott O. Chloride regulates afferent arteriolar contraction in response to depolarization. Hypertension. 1998;32 (6):1066-1070. doi:10.1161/01.HYP.32.6.1066

23. Ueda Y. Do we care about chloride? Proceedings of the 2017 International Veterinary Emergency and Critical Care Symposium, 14 Sep 2017. Nashville, USA

24. Magalhaes PA, de Brito TS, Freire RS, et al. Metabolic acidosis aggravates experimental acute kidney injury. Life Sci. 2016;146:48-65. doi:10.1016/j.lfs.2016.01.007

25. Davis JW, Park SN, Kaups KL, et al. Admission base deficit predicts transfusion requirements and risk of complications. $J$ Trauma. 1996;41(5):769-774. doi:10.1097/00005373-199611000-00001

26. Dunham CM, Siegel JH, Weireter L, et al. Oxygen debt and metabolic acidemia as quantitative predictors of mortality and severity of ischemic insult in hemorrhagic shock. Crit Care Med. 1991;19 (2):231-243. doi:10.1097/00003246-199102000-00020

27. Smith I, Kumar P, Molloy S, et al. Base excess and lactate as prognostic indicators for patients admitted to intensive care. Intensive Care Med. 2001;27(1):74-83. doi:10.1007/s001340051352

28. Hopper K, Haskins SC. A case-based review of a simplified quantitative approach to acid-base analysis. J Vet Emerg Crit Care. 2008;18 (5):467-476. doi:10.1111/j.1476-4431.2008.00344.x

29. Toyonaga Y, Kikura M. Hyperchloremic acidosis is associated with acute kidney injury after abdominal surgery. Nephrology. 2017;22 (9):720-727. doi:10.1111/nep. 12840

30. Zhang Z, Xu X, Fan H, et al. Higher serum chloride concentrations are associated with acute kidney injury in unselected critically ill patients. BMC Nephrol. 2013;14(1):235. doi:10.1186/1471-2369-14235

31. Gunnerson KJ. Clinical review: the meaning of acid-base abnormalities in the intensive care unit part 1 - epidemiology. Crit Care. 2005;9(5):508-516. doi:10.1186/cc3796

32. Gunnerson KJ, Saul M, He S, et al. Lactate versus non-lactate metabolic acidosis: a retrospective outcome evaluation of critically ill patients. Crit Care. 2006;10(1):R22. doi:10.1186/cc3987

33. Neyra JA, Canepa-Escaro F, Manllo J, et al. Association of hyperchloremia with hospital mortality in critically ill septic patients. Crit Care Med. 2015;43(9):1938-1944. doi:10.1097/CCM.0000000000 001161

34. Van Regenmortel N, Verbrugghe W, Van den Wyngaert T, et al. Impact of chloride and strong ion difference on ICU and hospital mortality in a mixed intensive care population. Ann Intensive Care. 2016;6(1):91. doi:10.1186/s13613-016-0193-x

35. Wehner A, Hartmann K, Hirschberger J. Associations between proteinuria, systemic hypertension and glomerular filtration rate in dogs with renal and non-renal diseases. Vet Record. 2008;162(5):141-147. doi:10.1136/vr.162.5.141

36. Surman S, Couto CG, Dibartola SP, et al. Arterial blood pressure, proteinuria, and renal histopathology in clinically healthy retired racing greyhounds. J Vet Intern Med. 2012;26(6):1320-1329. doi:10.1111/j.1939-1676.2012.01008.x

37. Littman MP, Daminet S, Grauer GF, IRIS Canine GN Study Group Diagnosis Subgroup, et al. Consensus recommendations for the diagnostic investigation of dogs with suspected glomerular disease. $J$ Vet Intern Med. 27;2013:S19-26. doi:10.1111/jvim.12223

38. Morales DL, Kavarana MN, Helman DN, et al. Restoration of renal function in shock by perfusion of the renal artery with venous blood: a counterintuitive approach. Crit. Care Med. 2002;30(6):1297-1300. doi:10.1097/00003246-200206000-00022 
39. Xie Z, Liao X, Yin W, et al. Relationship between short-term BP variability and incidence of AKI in critically ill patients. Kidney Blood Press Res. 2017;42(6):1238-1246. doi:10.1159/000485927

40. $\mathrm{Xu}$ JY, Ma SQ, Pan C, et al. A high mean arterial pressure target is associated with improved microcirculation in septic shock patients with previous hypertension: a prospective open label study. Crit Care. 2015;19(1):130. doi:10.1186/s13054-015-0866-0

41. Lee GT, Hwang SY, Jo IJ, et al. Associations between mean arterial pressure and 28-day mortality according to the presence of hypertension or previous blood pressure level in critically ill sepsis patients. J Thorac Dis. 2019;11(5):1980-1988. doi:10.21037/jtd.2019.04.108

42. Palaniappan L, Simons LA, Simons J, et al. Comparison of usefulness of systolic, diastolic, and mean blood pressure and pulse pressure as predictors of cardiovascular death in patients $\geq 60$ years of age (The dubbo study). Am J Cardiol. 2002;90(12):1398-1401. doi:10.10 16/S0002-9149(02)02884-9

43. Carlstrom M, Wilcox CS, Arendshorst WJ. Renal autoregulation in health and disease. Physiol Rev. 2015;95:405-511.36.

44. Loutzenhiser R, Griffin K, Williamson G, Bidani A. Renal autoregulation: new perspectives regarding the protective and regulatory roles of the underlying mechanisms. Am J Physiol Regul Integr Comp Physiol. 2005;290(5):R1153-67. doi:10.1152/ajpregu.00402.2005

45. Lefman SH, Prittie JE. Psychogenic stress in hospitalized veterinary patients: causation, implications, and therapies. J Vet Emerg Crit Care. 2019;29(2):107-120. doi:10.1111/vec.12821

46. Rose BD, Post TW. Metabolic acidosis. In: Clinical Physiology of Acid-Base and Electrolyte Disorders. 5th ed. New York: McGraw Hill; 2001:612-627.

47. Semler MW, Self WH, Wanderer JP, et al. Balanced crystalloids versus saline in critically ill adults. $N$ Engl $J$ Med. 2018;378 (9):829-839. doi:10.1056/NEJMoa1711584

48. Self WH, Semler MW, Wanderer JP, et al. Balanced crystalloids versus saline in noncritically ill adults. $N$ Engl $J$ Med. 2018;378 (9):819-828. doi:10.1056/NEJMoa1711586

49. Raghunathan K, Shaw AD, Nathanson B, et al. Association between the choice of IV crystalloid and in-hospital mortality among critically ill adults with sepsis. Crit Care Med. 2014;42(7):1585-1591. doi:10.1097/CCM.0000000000000305
50. Payen D, de Pont A-CJM, Sakr Y, et al. A positive fluid balance is associated with a worse outcome in patients with acute renal failure. Crit Care. 2008;12(3):R74.9. doi:10.1186/cc6916

51. Prittie J, Cazzolli D. Crystalloids versus colloids. In: Drobatz KJ, Hopper K, Rozanski E, Silverstein DC, editors. Textbook of Small Animal Emergency Medicine, I\&II. 1st ed. Wiley-Blackwell; 2018:1103-1114.

52. Cavanagh AA, Sullivan LA, Hansen BD. Retrospective evaluation of fluid overload and relationship to outcome in critically ill dogs. J Vet Emerg Crit Care. 2016;26(4):578-586. doi:10.1111/vec.12477

53. Bagshaw SM, Brophy PD, Cruz D, et al. Fluid balance as a biomarker: impact of fluid overload on outcome in critically ill patients with acute kidney injury. Crit Care. 2008;12(4):169. doi:10.1186/cc6948

54. Holte K, Sharrock NE, Kehlet H. Pathophysiology and clinical implications of perioperative fluid excess. Br J Anaesth. 2002;89 (4):622-632. doi:10.1093/bja/aef220

55. Barmparas G, Liou D, Lee D, et al. Impact of positive fluid balance in critically ill surgical patients: a prospective observational study. J Crit Care. 2014;29(6):936-941. doi:10.1016/j.jcrc.2014.06.023

56. Acheampong A, Vincent JL. A positive fluid balance is an independent prognostic factor in patients with sepsis. Crit Care. 2015;19 (1):251. doi:10.1186/s13054-015-0970-1

57. Bouchard J, Soroko SB, Chertow GM, et al. Fluid accumulation, survival and recovery of kidney function in critically ill patients with acute kidney injury. Kidney Int. 2009;76(4):422-427. doi:10.10 38/ki.2009.159

58. Giunti M, Troia R, Bergamini PF, Dondi F. Prospective evaluation of the acute patient physiologic and laboratory evaluation score and an extended clinicopathological profile in dogs with systemic inflammatory response syndrome. J Vet Emerg Crit Care. 2015;25(2):226-233. doi: $10.1111 /$ vec. 12257

\section{Publish your work in this journal}

Veterinary Medicine: Research and Reports is an international, peerreviewed, open access journal publishing original research, case reports, editorials, reviews and commentaries on all areas of veterinary medicine. The manuscript management system is completely online and includes a very quick and fair peer-review system. Visit http://www.dovepress.com/testimonials.php to read real quotes from published authors.

Submit your manuscript here: http://www.dovepress.com/veterinary-medicine-research-and-reports-journal 